Jurnal Ilmiah Matematika dan Pendidikan Matematika (JMP)

Vol. 11 No. 2, Desember 2019, hal. 1-10

ISSN (Cetak) : 2085-1456; ISSN (Online) : 2550-0422

\title{
A MULTIDIMENSIONAL SUBDIFFUSION MODEL WITH CHEMOTAXIS
}

\section{Bambang Hendriya Guswanto}

Analysis and Algebra Laboratory, Department of Mathematics

Faculty of Mathematics and Natural Sciences, Jenderal Soedirman University

Purwokerto 53123, Indonesia

Email : bambang.guswanto@unsoed.ac.id

\begin{abstract}
The mathematical model for subdiffusion process with chemotaxis proposed by Langlands and Henry [1] for the one-dimensional case is extended to the multidimensional case. The model is derived from random walks process using a probability measure on a n-multidimensional unit ball $S^{n-1}$.
\end{abstract}

Keywords: multi-dimensional model, subdiffusion, chemotaxis.

ABSTRAK. Model matematika untuk proses subdifusi dengan kemotaksis yang diturunkan oleh Langlands dan Henry [1] untuk kasus satu dimensi diperluas ke kasus multidimensi. Model ini diturunkan dari proses gerak acak dengan menggunakan ukuran peluang pada bola satuan multidimensi- $n S^{n-1}$.

Kata kunci: model multidimensi, subdifusi, kemotaksis.

\section{Introduction}

Consider the chemotaxis-diffusion system

$$
\begin{aligned}
& \frac{\partial u}{\partial t}=\Delta u-\nabla \cdot u \nabla v, \text { in } \Omega \times(0, \infty), \\
& \frac{\partial v}{\partial t}=\Delta v-v+u, \text { in } \Omega \times(0, \infty), \\
& \frac{\partial u}{\partial n}=\frac{\partial v}{\partial n}, \text { on } \partial \Omega \times(0, \infty), \\
& u(\cdot, 0)=u_{0}, \quad v(\cdot, 0)=v_{0}, \text { in } \Omega,
\end{aligned}
$$

where $\Omega \subset \mathbb{R}^{n}$ is a bounded domain with $C^{2}$ boundary. The System Eq. (1)-(4) is well known as the Keller-Segel Chemotaxis (KS) model. In this model (KS), $u$ and $v$ stand for the concentration of amobae and acrasin, respectively, where acrasin is a chemoattractant produced by the amobae. The model (KS) describes the space and time evolution of the concentration of diffusing amoebae that is chemotactically attracted by diffusing acrasin (see [2]). In general, the model (KS) can be used to explain the space and time evolution of the concentration of a diffusing species that is chemotactically attracted by a diffusing chemoattractant. 
Langlands and Henry [1] derived an equation describing the process in which the attracted species moves subdiffusively, which is called by the fractional chemotaxis-diffusion equation for the one-dimensional case. It is different from Eq. (1) describing the process in which the attracted species moves diffusively. Here, we extend the equation proposed by Langlands and Henry [1] for the onedimensional case to the multi-dimensional case. A particle moving in diffusion process follows the pattern

$$
\left\langle x^{2}(t)\right\rangle \sim t, t>0
$$

where $\left\langle x^{2}(t)\right\rangle$ is the mean square displacement at time $t>0$, whereas a particle moving in subdiffusion process follows the pattern

$$
\left\langle x^{2}(t)\right\rangle \sim t^{\alpha}, \quad 0<\alpha<1 .
$$

It means that the particle movement in subdiffusion process is slower than that in diffusion process (see[3]).

This paper is composed of three sections. In the second section, we introduce briefly the fractional integration and differentiation of Capuo operator. In the last section, our main result is shown.

\section{Fractional Time derivative}

Let $0<\alpha<1$ and $I=(0, T)$ for some $T>0$. The fractional integral of order $\alpha$ is defined by

$$
\frac{d^{-\alpha}}{d t^{-\alpha}} g(t)=\int_{0}^{t} \frac{(t-\tau)^{\alpha-1}}{\Gamma(\alpha)} g(\tau) d \tau, g \in L^{1}(T), \quad t>0 .
$$

For $\alpha=0$, we set $d^{-\alpha} / d t^{\alpha} g(t)=g(t)$. The fractional integral operator in Eq. (6) obeys the semigroup property

$$
\frac{d^{-\alpha}}{d t^{-\alpha}} \frac{d^{-\beta}}{d t^{-\beta}} g(t)=\frac{d^{-(\alpha+\beta)}}{d t^{-(\alpha+\beta)}} g(t), \quad 0 \leq \alpha, \beta<1
$$

The Caputo fractional derivative of order $\alpha$ is defined by

$$
\frac{d^{\alpha}}{d t^{\alpha}} g(t)=\int_{0}^{t} \frac{(t-\tau)^{-\alpha}}{\Gamma(1-\alpha)} \cdot \frac{d}{d \tau} g(\tau) d \tau, g \in W^{1,1}(T), t>0,
$$


where $*$ denotes the convolution of function

$$
(g * h)(t)=\int_{0}^{t} g(t-\tau) h(\tau) d \tau, \quad t>0
$$

and $W^{1,1}(I)$ is the set of all functions $u \in L^{1}(I)$ such that the distributional derivative of $u$ exists and belongs to $L^{1}(I)$. The operator $d^{\alpha} / d t^{\alpha}$ is a left inverse of $d^{-\alpha} / d t^{-\alpha}$, that is

$$
\frac{d^{\alpha}}{d t^{\alpha}} \frac{d^{-\alpha}}{d t^{-\alpha}} g(t)=g(t), \quad t>0
$$

but it is not a right inverse, that is

$$
\frac{d^{\alpha}}{d t^{\alpha}} \frac{d^{-\alpha}}{d t^{-\alpha}} g(t)=g(t)-g(0), t>0
$$

We next introduce Mittag-Leffler function defined by

$$
E_{\alpha, \beta}(z)=\sum_{j=0}^{\infty} \frac{z^{j}}{\Gamma(\alpha j+\beta)}, \quad \alpha, \beta>0, \quad z \in \mathbb{C} .
$$

This function is entire. For $\beta=1$, we set $E_{\alpha, \beta}(z)=E_{\alpha}(z)$ and, for $\alpha=\beta=1$, we get that $E_{\alpha, \beta}(z)$ is nothing but exponential function $e^{z}$. We refer to Kilbas et al. [4] and Podlubny [5] for more details concerning the fractional integral and derivative.

\section{Main Results}

We consider a particle moving in a random walk process. In this process, we suppose $T(x, t ; \omega), \phi(t)$, and $q(x, t)$ stand for the jump probability of the particle in the direction $\omega \in S^{n-1}$ from position $x$ at time $t$, the waiting time probability of the particle to jump after waiting a time $t>0$, and the concentration of the particles at position $x$ at time $t$, respectively, where $S^{n-1}=$ $\left\{\omega \in \mathbb{R}^{n}:|\omega|=1\right\}$. Following Langlands and Henry [2], we start from the master equation

$$
\begin{aligned}
q(x, t)= & q(x, 0) \Phi(t) \\
& +\int_{0}^{t} \int_{S^{n-1}} \phi(t-\tau) T(x-\omega \Delta x, \tau ; \omega) q(x-\omega \Delta x, \tau) d \omega d x .
\end{aligned}
$$


to derive our model in the multidimensional case. The equation means that the concentration of the particles at position $x$ at time $t$ consists of the concentration of the particles at position $x$ at time $t=0$ which have not yet jumped (the first term on the right hand side) and the concentration of the particles at position $x-\omega \Delta x$ at an earlier time $\tau$ that then jumps at time $t$ after waiting a time $t-\tau$ in the direction $\omega$ with the constant jump length $\Delta x$ (the second term on the right hand side). Using Laplace transform applied to Eq. (9), we have

$$
\begin{aligned}
\tilde{q}(x, s)= & \delta(x)\left(\frac{1-\tilde{\phi}(s)}{s}\right) \\
& +\tilde{\phi}(s) \int_{s^{n-1}} \tilde{T}(x-\omega \Delta x, s ; \omega) \tilde{q}(x-\omega \Delta x, s) d \omega
\end{aligned}
$$

where

$$
\tilde{h}(s)=\int_{0}^{\infty} e^{-s x} h(x) d x .
$$

Consequently, using the invers of Laplace transform applied to Eq. (10), we get

$$
\begin{aligned}
q(x, t) & -q(x, 0) \\
& =\int_{0}^{t} H(t-\tau)\{-q(x, \tau) \\
& \left.+\int_{s^{n-1}} T(x-\omega \Delta x, \tau ; \omega) q(x-\omega \Delta x, \tau) d \omega\right\}
\end{aligned}
$$

where

$$
\widetilde{H}(s)=\frac{\tilde{\phi}(s)}{1-\tilde{\phi}(s)} .
$$

We now suppose that the jump probability $T$ is a constant or does not depend on the direction $\omega$ and time $t$. It can be interpreted that the particle in the process moves in a homogen medium or the particle movement is not influenced by any external force field. In this case, since $T$ satisfies the normalized condition 


$$
\int_{S^{n-1}} T(x, \tau ; \omega) d \omega=1
$$

then $T(x, \tau ; \omega)=1 /\left|S^{n-1}\right|$ where

$$
\left|S^{n-1}\right|=\int_{S^{n-1}} d \omega=\frac{2 \pi^{n / 2}}{\Gamma(n / 2)}
$$

It follows that Eq. (11) becomes

$$
\begin{aligned}
q(x, t) & -q(x, 0) \\
& =\frac{1}{\left|S^{n-1}\right|} \int_{0}^{t} H(t-\tau)\left\{\int_{s^{n-1}} q(x-\omega \Delta x, \tau)-q(x, \tau) d \omega\right\} .
\end{aligned}
$$

Lemma 1 ([6]). If $g=g(x)$ is a $C^{2}$ function then

$$
\int_{S^{n-1}} f(x+\omega \Delta x)-f(x) d \omega=\frac{1}{n}\left|S^{n-1}\right|(\Delta x)^{2} f(x)+o\left((\Delta x)^{2}\right)
$$

as $\Delta x \rightarrow 0$.

Applying Lemma 1 to Eq. (12), we obtain

$$
q(x, t)-q(x, 0)=\frac{(\Delta x)^{2}}{2 n} \int_{0}^{t} H(t-\tau) \Delta q(x, \tau) d \tau+o\left((\Delta x)^{2}\right)
$$

as $\Delta x \rightarrow 0$. When the random walk process is Markovian or the waiting time probability is Poissonian

$$
\phi(t)=\frac{1}{\lambda} e^{-\frac{t}{\lambda}}, t \geq 0, \lambda>0,
$$

by simple calculation, we get $H(t)=1 / \lambda$. Then, Eq. (13) is reduced to

$$
q(x, t)-q(x, 0)=\frac{(\Delta x)^{2}}{2 n \lambda} \int_{0}^{t} \Delta q(x, \tau) d \tau+o\left((\Delta x)^{2}\right)
$$

as $\Delta x \rightarrow 0$. Thus, if we take the limit $\Delta x \rightarrow 0, \lambda \rightarrow 0$, and $(\Delta x)^{2} / \lambda$ is kept finite then Eq. (15) becomes 


$$
\frac{\partial q}{\partial t}=D \Delta q
$$

where

$$
D=\frac{(\Delta x)^{2}}{2 n \lambda}
$$

Eq. (16) is well known as Diffusion equation, whereas Eq. (17) is called by Diffusion coefficient.

We now assume that the random walk process is non Markovian with the waiting time probability

$$
\phi_{\alpha}(t)=\frac{t^{\alpha-1}}{\lambda^{\alpha}} E_{\alpha, \alpha}\left(-\left(\frac{t}{\lambda}\right)^{\alpha}\right), 0<\alpha<1, \lambda>0, t>0 .
$$

The process with the waiting time probability expressed by Eq. (18) has the characteristic as described by Eq. (5) (see [7]). By Laplace transform and its invers, from Eq. (18), we obtain

$$
H(t)=\frac{1}{\lambda^{\alpha}} \cdot \frac{t^{\alpha-1}}{\Gamma(\alpha)}
$$

Then Eq. (13) is reduced to

$$
q(x, t)-q(x, 0)=\frac{(\Delta x)^{2}}{2 n \lambda^{\alpha}} \int_{0}^{t} \frac{(t-\tau)^{\alpha-1}}{\Gamma(\alpha)} \Delta q(x, \tau) d \tau+o\left((\Delta x)^{2}\right)
$$

as $\Delta x \rightarrow 0$. Consequently, by Eq. (8) and (19), we get

$$
\frac{\partial^{\alpha}}{\partial t^{\alpha}} q(x, t)=\frac{(\Delta x)^{2}}{2 n \lambda^{\alpha}} \Delta q(x, t)+o\left((\Delta x)^{2}\right)
$$

as $\Delta x \rightarrow 0$. Thus, if we take the limit $\Delta x \rightarrow 0, \lambda \rightarrow 0$, and $(\Delta x)^{2} / \lambda^{\alpha}$ is kept finite then Eq. (20) is reduced to

$$
\frac{\partial^{\alpha} q}{\partial t^{\alpha}}=D_{\alpha} \Delta q
$$

where

$$
D_{\alpha}=\frac{(\Delta x)^{2}}{2 n \lambda^{\alpha}} .
$$

Eq. (21) and (22) are called by Subdiffusion equation and coefficient, respectively.

We next suppose that $T$ satisfies 


$$
\begin{aligned}
& T(x, t ; \omega)-T(x, t ;-\omega)=F(x, t ; \omega), \\
& T(x, t ; \omega)+T(x, t ;-\omega)=G(x, t ; \omega),
\end{aligned}
$$

where $F \neq 0$, which means that the process is influenced by an external force field. For the case $F=0$, it is exactly same as that describing the process in which the particle moves in a homogen medium or the particle movement is not influenced by any external force field as explained previously. By the normalized condition of $T$, we have

$$
\frac{1}{2} \int_{S^{n-1}} G(x, t ; \omega)=1 .
$$

For the jump lenght $\Delta x$ sufficiently small, by Taylor-series expansion with respect to $x$, we next get

$$
\begin{aligned}
& T(x-\omega \Delta x, t ; \omega) q(x-\omega \Delta x, t) \\
&= T(x, t ; \omega) q(x, t)-\Delta x \omega \cdot \nabla T(x, t ; \omega) q(x, t) \\
&+\frac{1}{2}(\Delta x)^{2}\{\omega \cdot \nabla\}^{2} T(x, t ; \omega) q(x, t)+o\left((\Delta x)^{2}\right) .
\end{aligned}
$$

Similarly,

$$
\begin{aligned}
T(x+ & \omega \Delta x, t ; \omega) q(x+\omega \Delta x, t) \\
= & T(x, t ; \omega) q(x, t)+\Delta x \omega \cdot \nabla T(x, t ; \omega) q(x, t) \\
& +\frac{1}{2}(\Delta x)^{2}\{\omega \cdot \nabla\}^{2} T(x, t ; \omega) q(x, t)+o\left((\Delta x)^{2}\right) .
\end{aligned}
$$

By Eq. (25) and (26), we then obtain

$$
\begin{aligned}
& T(x-\omega \Delta x, t ; \omega) q(x-\omega \Delta x, t)+T(x+\omega \Delta x, t ; \omega) q(x+\omega \Delta x, t) \\
&= G(x, t ; \omega) q(x, t)-\Delta x \omega \cdot \nabla F(x, t ; \omega) q(x, t) \\
&+\frac{1}{2}(\Delta x)^{2}\{\omega \cdot \nabla\}^{2} G(x, t ; \omega) q(x, t)+o\left((\Delta x)^{2}\right) .
\end{aligned}
$$

We then suppose that $F$ satisfies the condition

$$
F(x, t ; \omega)=\frac{\Delta x}{\left|S^{n-1}\right|} \omega \cdot f(x, t),
$$

where $f(x, t)=\left(f_{1}(x, t), f_{2}(x, t), \cdots, f_{n}(x, t)\right) \in \mathbb{R}^{n}$ is the external force field influencing the particle jump at position $x$ at time $t$ and $f_{i}(x, t), i=1,2, \cdots n$, is the external force field influencing the particle jump at position $x$ at time $t$ in the 
$i^{\text {th }}$-direction of $n$-dimensional coordinate system. We also suppose that $G(x, t ; \omega)$ is a constant implying $G(x, t ; \omega)=2 /\left|S^{n-1}\right|$. Since

$$
\begin{aligned}
& \int_{S^{n-1}} \omega_{i} d \omega=0, \quad i=1,2, \cdots, n, \\
& \int_{S^{n-1}} \omega_{i} \omega_{j} d \omega=\delta_{i j} \frac{\left|S^{n-1}\right|}{n}, i, j=1,2, \cdots, n,
\end{aligned}
$$

where $\omega=\left(\omega_{1}, \omega_{2}, \cdots, \omega_{n}\right) \in S^{n-1}$, then, by Eq. (27),

$$
\begin{aligned}
-q(x, \tau) & +\int_{S^{n-1}} T(x-\omega \Delta x, \tau ; \omega) q(x-\omega \Delta x, \tau) d \omega \\
= & -q(x, \tau)+\frac{1}{2} \int_{S^{n-1}}[T(x-\omega \Delta x, \tau ; \omega) q(x-\omega \Delta x, \tau) \\
& +T(x+\omega \Delta x, \tau ; \omega) q(x+\omega \Delta x, \tau)] d \omega+o\left((\Delta x)^{2}\right) \\
= & -\frac{1}{2} \Delta x \int_{S^{n-1}} \omega \cdot \nabla F(x, t ; \omega) q(x, t) d \omega \\
& +\frac{1}{4}(\Delta x)^{2} \int_{S^{n-1}}\{\omega \cdot \nabla\}^{2} G(x, t ; \omega) q(x, t) d \omega+o\left((\Delta x)^{2}\right) .
\end{aligned}
$$

Thus, by Eq. (28), (29), and (30), Eq. (11) is reduced to

$$
\begin{aligned}
q(x, t) & -q(x, 0) \\
& =\frac{(\Delta x)^{2}}{2 n} \int_{0}^{t} H(t-\tau)\{\Delta q(x, \tau)-\nabla \cdot f(x, \tau) q(x, \tau)\} d \tau \\
& +o\left((\Delta x)^{2}\right) .
\end{aligned}
$$

Using the waiting time probabilities Eq. (14) and Eq. (18), Eq. (32) becomes

$$
\frac{\partial}{\partial t} q(x, t)=D \nabla \cdot(\nabla q(x, t)-f(x, \tau) q(x, \tau))
$$

and

$$
\frac{\partial^{\alpha}}{\partial t^{\alpha}} q(x, t)=D_{\alpha} \nabla \cdot(\nabla q(x, t)-f(x, \tau) q(x, \tau))
$$


respectively. Eq. (33) is well known as Fokker-Planck equation, whereas Eq. (34) is called as Fractional Fokker-Planck equation or Fokker-Planck equation with subdiffusive kinetics.

Following Stevens [8] for the multi-dimensional case, we now suppose that the jump probability in the direction $\omega$ depends on the chemoattractant on a side of the current position, that is

$$
T(x, t ; \omega)=\frac{v(x+\Delta x \omega, t)}{\int_{S^{n-1}} v(x-\Delta x \omega, t) d w}
$$

where $v(x, t)$ is a sensitivity function depending on the concentration $c(x, t)$ of the chemoattractant at position $x$ at time $t$

$$
v(x, t)=e^{\gamma c(x, t)} \text {. }
$$

Assuming $G(x, t ; \omega)$ is a constant, we then have

$$
\int_{S^{n-1}} v(x-\Delta x \omega, t) d w=\frac{\left|S^{n-1}\right|}{2}[v(x+\Delta x \omega, t)+v(x-\Delta x \omega, t)] .
$$

Consequently,

$$
\begin{aligned}
T(x, t ; \omega)-T(x, t ; \omega) & =\frac{v(x+\Delta x \omega, t)-v(x-\Delta x \omega, t)}{\int_{S^{n-1}} v(x-\Delta x \omega, t) d w} \\
& =\frac{2}{\left|S^{n-1}\right|} \cdot \frac{e^{\gamma c(x+\Delta x \omega, t)}-e^{\gamma c(x-\Delta x \omega, t)}}{e^{\gamma c(x+\Delta x \omega, t)}+e^{\gamma c(x-\Delta x \omega, t)}} \\
& \approx \frac{2}{\left|S^{n-1}\right|} \cdot \frac{e^{\gamma \Delta x \omega \cdot \nabla c(x, t)}-e^{-\gamma \Delta x \omega \cdot \nabla c(x, t)}}{e^{\gamma \Delta x \omega \cdot \nabla c(x, t)}+e^{-\gamma \Delta x \omega \cdot \nabla c(x, t)}} \\
& =\frac{2}{\left|S^{n-1}\right|} \tanh (\gamma \Delta x \omega \cdot \nabla c(x, t)) \\
& \approx 2 \gamma \frac{\Delta x}{\left|S^{n-1}\right|} \omega \cdot \nabla c(x, t) .
\end{aligned}
$$

Thus, by Eq. (23), (24), (33), (34), (36) and using Eq. (14) and (18) again, we obtain

$$
\frac{\partial}{\partial t} q(x, t)=D \Delta q(x, t)-\chi \nabla \cdot q(x, t) \nabla c(x, t)
$$

and 


$$
\frac{\partial^{\alpha}}{\partial t^{\alpha}} q(x, t)=D_{\alpha} \Delta q(x, t)-\chi_{\alpha} \nabla \cdot q(x, t) \nabla c(x, t),
$$

respectively, where

$$
\chi=\frac{\gamma(\Delta x)^{2}}{n \lambda}, \quad \chi_{\alpha}=\frac{\gamma(\Delta x)^{2}}{n \lambda^{\alpha}} .
$$

Eq. (37) is well known as the mathematical model of chemotaxis-diffusion describing the space and time evolution of the concentration of a particle that moves diffusively and is chemotactically attracted by a diffusing chemoattractant, whereas Eq. (38) is the mathematical model for chemotaxis-diffusion process in which the particle involved moves subdiffusively and is chemotactically attracted by a diffusing chemoattractant.

\section{References}

[1] Langlands, T. A. M. \& Segel, L. A., Fractional Chemotaxis Diffusion Equations, Physical Review E 81, 0511022010.

[2] Keller, E. F. \& Segel, L. A., Initiation of Slime Mold Agregation Viewed as an Instability, J. Theor. Biol., 26(1970), pp. 399-415.

[3] Metzler, R. \& Klafter, J., The Random Walk's Guide to Anomalous Diffusion : Fractional Dynamics Approach, Physics Report, 339, pp. 1-77, 2005.

[4] Kilbas, A. A., Srivastava, H. M., \& Trujilo, J. J., Theory and Applications of Fractional Differential Equations, North Holland Mathematics Studies, Elsevier, 2006.

[5] Podlubny, I., Fractional Differential Equations, Academic Pres, 1999.

[6] Senba, T. \& Suzuki, T., Applied Analysis : Mathematical Methodes in Natural Sciences, Ed. 2, Imperial College Press, 2010.

[7] Gorenflo, R., Mittag-Leffler Waiting Time, Power laws, Rarefaction, Continuous Random Walk, Diffusion Limit, Proceedings of the National Workshop on Fractional Calculus and Statistical Distributions, pp. 1-22, 2009.

[8] Stevens, A., A Stochastic Cellular Automation Modeling Gliding and Agregation of Myxobacteria, Siam J. Appl. Math., 61(1), 172-182, 2000. 